LAWRENCE LIVERMORE NAT IO N A L LABORATORY

\section{An Integrated Universal Collapsar Gamma-ray Burst Model}

Jay D. Salmonson

January 22, 2004

Gamma-ray Burst Symposium

Santa Fe, NM, United States

September 8, 2003 through September 12, 2003 
This document was prepared as an account of work sponsored by an agency of the United States Government. Neither the United States Government nor the University of California nor any of their employees, makes any warranty, express or implied, or assumes any legal liability or responsibility for the accuracy, completeness, or usefulness of any information, apparatus, product, or process disclosed, or represents that its use would not infringe privately owned rights. Reference herein to any specific commercial product, process, or service by trade name, trademark, manufacturer, or otherwise, does not necessarily constitute or imply its endorsement, recommendation, or favoring by the United States Government or the University of California. The views and opinions of authors expressed herein do not necessarily state or reflect those of the United States Government or the University of California, and shall not be used for advertising or product endorsement purposes. 


\title{
An Integrated Universal Collapsar Gamma-ray Burst Model
}

\author{
Jay D. Salmonson \\ Lawrence Livermore National Laboratory, Liveremore, CA 94551
}

\begin{abstract}
.
Starting with two assumptions: (1) gamma-ray bursts originate from stellar death phenomena or so called "collapsars" and (2) that these bursts are quasi-universal, whereby the majority of the observed variation is due to our perspective of the jet, an integrated gamma-ray burst model is proposed. It is found that several of the key correlations in the data can be naturally explained with this simple picture and another possible correlation is predicted.
\end{abstract}

\section{INTRODUCTION}

A wealth of data is continuing to be accrued that indicates gamma-ray bursts derive from stellar death events. To date there are two certain directly observed associations of a GRB with a type Ib/c supernova: GRB980425 with sn1998bw [1] and GRB030329 with sn2003dh[2]. In several other cases a ruddy bump[e.g. 3, 4], ostensibly the underlying supernova, has been observed in the decaying GRB afterglow lightcurves. These direct indications, coupled with the fact that GRBs tend to originate in star forming regions [5, 6], seem to indicate that GRB explosions are the product of a stellar death event which also produces a supernova - so called "collapsars"[7].

At the same time evidence is emerging to indicate that cosmological gamma-ray bursts might be quasi-universal in that the innate variation from burst to burst might be of order a factor of two or so, while the observed variation of fluxes and timescales can be as much as two orders of magnitude. In the same spirit of the unified models for active galactic nuclei, the wide range of observed GRB quantities is dependent on the observer viewing angle with respect to a single, universal structured jet.

Herein I simply combine these two proposed features of GRBs and attempt to formulate an integrated model. It is found that by literally interpreting the hydrodynamic nature of the emerging jet from a collapsar, as shown from numerical simulations, it is relatively natural to be able to broadcast material into the range of angles and energies inferred from the correlations in the data. A model is sketched out which nominally satisfies the key correlations in the data. As such, this model provides an integrated picture of what a universal collapsar GRB might look like. 


\section{THE MODEL}

Begin with a collapsar. Numerical simulations[8, 9] demonstrate that introducing an energy source at the center of a collapsing star can produce an energetic jet that bores along the rotation axis of the star and erupts from the surface intact, thereby blowing up the star in the process. They also demonstrate modest Lorentz factors, $\gamma \sim 10-20$ of the jet as it emerges from the star, with a similar internal energy per rest mass $\eta \sim 10-20$.

If GRBs derive from collapsars, I argue that, regardless of the nature of the source of energy at the core of the star, these simulations must be qualitatively correct. Therefore, the first assumption of this model is that a relativistic jet emerging from a star is hydrodynamic in nature; the second law of thermodynamics virtually ensures that shocks, instabilities, magnetic reconnections will render the emerging material hot and with high entropy. Furthermore, I make the plausible assumption, inspired by said jet simulations, that the energy of the emerging jet material will be roughly equally partitioned between kinetic energy and internal energy: $\gamma \approx \eta$.

As such, when a parcel of jet material emerges from the star, no longer being confined, it will explode. This explosion must be isotropic in the co-moving frame of the material and will blow it into a thin spherical shell with terminal Lorentz factor equal to $\eta$. In the lab frame the parcel emerged from the star with Lorentz factor, $\gamma$, therefore the bulk of the energy in the shell will be beamed into an angle $1 / \gamma$ (see Fig. 1). Still in the lab frame, the terminal Lorentz factor of the exploded shell moving along the jet axis will be $\Gamma \sim \gamma \eta$ and, since $\gamma \approx \eta$, will vary from $\Gamma \approx 2 \gamma^{2}$ along the jet axis, to $\Gamma \approx \gamma^{2}$ at an angle $1 / \gamma$ from the jet axis. So in this model $1 / \gamma$ is angular scale and $\Gamma \propto \gamma^{2}$ is the specific energy (i.e. Lorentz factor) scale. This secondary acceleration scenario has also been proposed in the "firework" model of GRBs from highly magnetized black holes [10].

An immediate consequence of this model is a simple correspondence between opening angle, $\theta$, and Lorentz factor, $\Gamma: \theta \propto 1 / \gamma \propto 1 / \sqrt{\Gamma}$. This provides a direct correspondence between viewing angles, as inferred from afterglow jet-break times, and the Lorentz factors of the GRB prompt emission. These viewing angles range from $3^{\circ}$ to $20^{\circ}$, implying a range of $\gamma$ from 20 to 3 [11]. Assuming $\eta \approx \gamma$, the final Lorentz factor $\Gamma$ will range from $2 \gamma \eta$ to $\gamma \eta$, or from 800 to 9 .

It is an intriguing correspondence that this range of Lorentz factors, from $\sim 1000$ for the brightest bursts to $\sim 10$ for the dimmest, is consistent with that found by using kinematic arguments [12] and assuming GRB980425 was only mildly relativistic, $\Gamma_{980425} \sim 2$. Furthermore, several workers[13, 14] have independently given $\Gamma_{990123} \sim$ 1000 , one of the brightest burst ever observed. In fact it might be telling that the lower end of this range, $\Gamma \sim 10$, is about the lower limit that compactness of the source can allow for optically thin, non-thermal emission [15]. Such a range of Lorentz factors, ranging over a factor of $\sim 100$ is perhaps unorthodox, however, given that luminosities, timescales and energies span this range, I argue that such variation in Lorentz factor is, at least, plausible.

So in this model the jet that emerges from the collapsar has a variable Lorentz factor, $3 \lesssim \gamma(t) \approx \eta(t) \lesssim 20$ which, by secondary expansion, generates a sequence of concentric expanding shells, each with its own terminal Lorentz factor, $10 \lesssim \Gamma \lesssim 1000$, and opening 

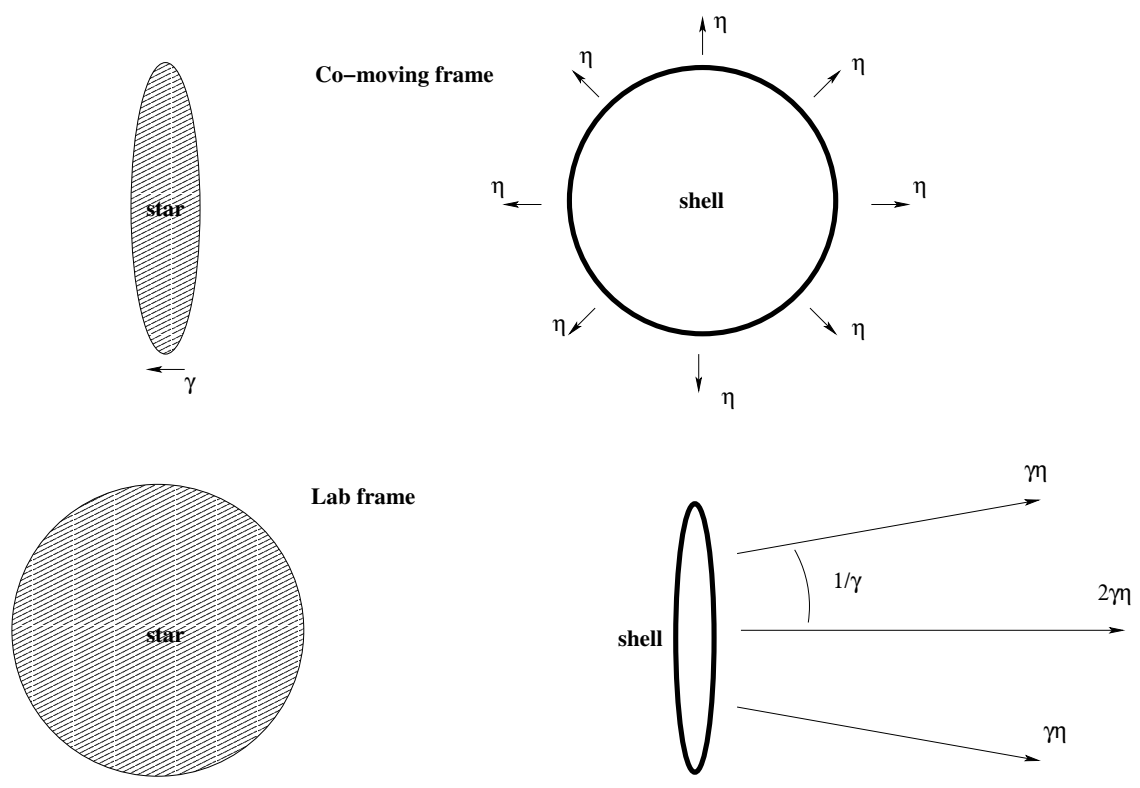

FIGURE 1. A schematic representation of a blob of material emitted from a dying star with Lorentz factor $\gamma$ and specific internal energy $\eta$. No longer confined by the star, the blob expands isotropically, in its co-moving frame, into a shell moving with terminal Lorentz factor $\eta$. In the lab frame this material is boosted to a Lorentz factor $\sim \gamma \eta$, but with a characteristic beaming angle $1 / \gamma$.

angle, $20^{\circ} \gtrsim \theta \sim 1 / \gamma \gtrsim 3^{\circ}$. The subsequent collision of these shells creates a viewing angle dependent variety of GRBs and the interaction with the interstellar medium creates a viewer angle dependent afterglow.

\section{COMPARISON WITH THE CORRELATIONS}

An intriguing property of GRBs and their afterglows that has emerged in the last few years is that, save a few interesting exceptions, they tend to align along several simple correlations. The existence of simple monotonic relationships of several key observable energy and timescales strongly implies a single variable that governs the observed variation. With this realization it becomes an intriguing possibility that all bursts are, to first order, quite similar and that the dominant contributor to the observed diversity is observer angle with respect to the burst jet axis. This paradigm has the added benefit of identifying the expected variation with viewing angle as being the source of the observed breadth of the correlations. As described in the following, the integrated universal collapsar model outlined here can describe, or consistently draw inferences from, most of the existing correlations.

$\checkmark E_{\gamma} \propto$ constant The fact that the majority of cosmological GRBs seem to have a constant total energy in gamma-rays[11, 16], despite the wide variation in their luminosities and timescales, is an intriguing and mysterious clue about the progenitor. However, this mysterious clue becomes a prosaic and even trivial matter of perspective if the energy per steradian of the afterglow shock varies as $\varepsilon \propto \theta^{-2}$ 
with respect to the jet axis[17, 18]. Thus the observed variety stems merely from the variety of observer angles on a universal jet. If one takes this afterglow energy distribution as given, then one can derive the implied energetics of the emerging fireball. This gives that the energy and mass distributions of the emitted blobs are

$$
\frac{d \varepsilon}{d \gamma} \propto \gamma, \quad \frac{d M}{d \gamma} \propto 1 / \gamma .
$$

The implication that the most energetic material is the least mass loaded is very plausible; the lightest material moves the fastest. There is no obvious physical reason that these scalings be exact and they are likely approximate.

$\checkmark L \propto \Delta t^{-1}$ One of the earliest discovered correlations is that of peak luminosity, $L$, with the reciprocal temporal lag, $\Delta t$, of lower energy gamma-rays behind their higher energy counterparts within a prompt emission pulse [19]. A kinematic interpretation[20] simply states that luminosity will scale with Lorentz factor and temporal lag will vary inversely with Lorentz factor[see also Sec. 3 of ref. 21]. In this mode observers nearer the jet axis will see higher velocity material and thus larger luminosities and shorter lags.

$\checkmark L \propto v a r i a b i l i t y$ The trend that more luminous bursts tend to be more variable, with more, and narrower, spikes[22, 23] has a natural explanation in this model. As with the lag-luminosity relationship discussed above, the highest energy shells will have their energy confined more closely along the jet axis, and thus the more energetic collisions will radiate more closely along the jet axis. Thus observers near the jet axis will observe more collisions, therefore more variability, than those at higher viewing angles.

$\checkmark \Delta t \propto t_{j} \quad$ A surprisingly tight connection between the prompt gamma-ray phase and subsequent afterglow phase[21] is seen by this linear correlation between the temporal lag, $\Delta t$, of lower energy gamma-rays behind their higher energy counterparts within a prompt emission pulse, and the jet-break time, $t_{j}$, at which the afterglow temporal power-law decay index steepens. This correlation is demonstrable by noting $t_{j} \propto \varepsilon^{1 / 3} \theta^{8 / 3} \propto \theta^{2} \propto \gamma^{-2} \propto \Gamma^{-1}$, where $\varepsilon \propto \theta^{-2}$ from the structured jet, and $\Delta t \propto \Gamma^{-1}$ from the kinematics of the lag-luminosity relationship, so $t_{j} \propto \Delta t$.

$\times E_{p k} \propto \sqrt{E_{i s o}}$ A clue to the gamma-ray emission mechanism is that the peak of the spectral energy distribution, $E_{p k}$, varies like the square root of the inferred isotropic burst energy, $E_{\text {iso }}[24,25]$. This relationship is quite enigmatic. It is the opinion of the author that until the nature of the emission mechanism is better understood, this correlation remains unexplained within the context of this model or any other. Future work will address this issue.

- Prediction: $t_{d e c} \propto \theta^{14 / 3} \quad$ A prediction of this model is that there should be a rather strong dependence of the deceleration time, $t_{d e c}$, which roughly corresponds to the commencement of the forward shock afterglow emission, on viewing angle, $\theta$. Specifically, $t_{d e c} \propto\left(\varepsilon \Gamma^{-8}\right)^{1 / 3} \propto \Gamma^{-7 / 3} \propto \theta^{14 / 3}$. Thus the expected range of afterglow onsets should be $\left(20^{\circ} / 3^{\circ}\right)^{14 / 3} \sim$ seconds per day. Therefore, given the very prompt, $\sim 1$ second, onset of the afterglow and attendant reverse shock of 
bright burst such as 990123, one would expect an onset of the external shock afterglow for weaker bursts to be seen at $\sim 1$ day, which is precisely what is observed [e.g. 970508, 26].

The model introduced here is very coarse. However, it does describe several of the key correlations encompassing a broad range of physical observables, and suggests another, in the context of a collapsar model by simply and naturally employing the result of numerical simulations that the jet eruption is hydrodynamic. This logically and directly leads to the idea of secondary acceleration and expansion of the equipartition heated gas emerging from the star. Indeed, if GRBs both derive from collapsars and are quasiuniversal, then it is highly plausible that at least some of the key features described here must come into play. It is interesting that the picture of the gamma-ray burst jet described here is qualitatively different than what has been described in the literature to date.

\section{ACKNOWLEDGMENTS}

I wish to thank E. Ramirez-Ruiz, S. Woosley and A. MacFadyen for useful discussions regarding this work. This work was performed under the auspices of the U.S. Department of Energy by University of California Lawrence Livermore National Laboratory under contract W-7405-ENG-48.

\section{REFERENCES}

1. Galama, T. J. et al. Nature, 395, 670-672 (1998).

2. Stanek, K. Z. et al. ApJL, 591, L17-L20 (2003).

3. Bloom, J. S. et al. Nature, 401, 453-456 (1999).

4. Reichart, D. E., ApJ, 554, 643-659 (2001).

5. Hogg, D. W., and Fruchter, A. S., ApJ, 520, 54-58 (1999).

6. Bloom, J. S., Kulkarni, S. R., and Djorgovski, S. G., AJ, 123, 1111-1148 (2002).

7. MacFadyen, A. I., and Woosley, S. E., ApJ, 524, 262-289 (1999).

8. Zhang, W., Woosley, S. E., and MacFadyen, A. I., ApJ, 586, 356-371 (2003).

9. Aloy, M. A., Müller, E., Ibáñez, J. M., Martí, J. M., \& MacFadyen, A. 2000, ApJL, 531, L119

10. Barbiellini, G., Celotti, A., and Longo, F., MNRAS, 339, L17-L21 (2003).

11. Frail, D. A. et al. ApJL, 562, L55-L58 (2001).

12. Salmonson, J. D., ApJL, 546, L29-L31 (2001).

13. Panaitescu, A., and Kumar, P., ApJ, 554, 667-677 (2001).

14. Soderberg, A. M., and Ramirez-Ruiz, E., AIP Conference Series 662, 2003, pp. 172-175.

15. Piran, T., Phys. Rep., 333, 529-553 (2000).

16. Bloom, J. S., Frail, D. A., and Kulkarni, S. R., ApJ, 594, 674-683 (2003).

17. Rossi, E., Lazzati, D., and Rees, M. J., MNRAS, 332, 945-950 (2002).

18. Zhang, B., and Mészáros, P., ApJ, 571, 876-879 (2002).

19. Norris, J. P., Marani, G. F., and Bonnell, J. T., ApJ, 534, 248-257 (2000).

20. Salmonson, J. D., ApJL, 544, L115-L117 (2000).

21. Salmonson, J. D., and Galama, T. J., ApJ, 569, 682-688 (2002).

22. Ramirez-Ruiz, E., and Fenimore, E., astro-ph/0004176 (2000).

23. Reichart, D. E. et al. ApJ, 552, 57-71 (2001).

24. Lloyd, N. M., Petrosian, V., and Mallozzi, R. S., ApJ, 534, 227-238 (2000).

25. Amati, L. et al. AAP, 390, 81-89 (2002).

26. Pian, E. et al. ApJL, 492, L103+ (1998). 